\title{
TCP throughput over links with high Bit Error Rate
}

\author{
Tanja Lang, Daniel Floreani and Arek Dadej \\ Cooperative Research Center for Satellie Systems Institute for Telecommunications Research, \\ University of South Australia
}

\begin{abstract}
We investigated the throughput performance of different versions of TCP for different bit error rates and different round trip times. A new modular TCP implementation was used for the study that allowed us to combine any congestion control mechanism with any error detection strategy. The goal of our study was to find the combination that provides the highest throughput in a high BER environment. We found that no particular protocol variant performed considerable better for all BERs. The most interesting result of our study was that the error detection strategy developed for TCP NewReno performs as well as selective acknowledgments for most BERs and RTTs.
\end{abstract}

Key words: TCP performance, high BER, congestion control, error recovery

\section{INTRODUTCTION}

As the Internet is developing, the use of non-wireline transmission media becomes increasingly common. Mobile users want to connect to the Internet using wireless technology, companies and universities extend their facilities with wireless local area networks and satellites are used to connect users in remote geographical areas or to interconnect distant network islands.

These new underlying network technologies have quite different physical properties to the commonly used Ethernet or optical cables. A connection running over these new technologies can experience one or more of the following network path characteristics: Scarce bandwidth, high or variable propagation delay, high or variable access delay due to medium access protocols, handovers, intermittent connectivity, packet loss due to channel noise or asymmetric forward and return channel bandwidth. 
In recent years, many research projects have focused on evaluating the traditional internetworking protocols in these heterogeneous environments ([1]-[3]). These projects have shown that the new operating environments especially impact the Transmission Control Protocol (TCP), the most commonly used protocol for reliable data transfer. High delays, high Bit Error Rate (BER), high asymmetry and intermittent connectivity all pose problems for TCP's reliability mechanisms.

As part of the co-operative research centre for satellite systems, we are running a project to evaluate TCP in these new environments. In this paper, we present our investigation on the impact of high BER on TCP throughput. Previously we have evaluated the impact of high propagation delay on TCP ([4]).

High BER presents two challenges to the reliable TCP protocol. The first one is for the congestion control mechanism. Packet drops due to corruption violate TCP's assumption that all packet losses are caused by congestion. In non-wireless networks, for which TCP was developed, congestion is the main cause of packet loss. Therefore, TCP slows down its transmission rate whenever a packet loss is detected to reduce the congestion in the network. This slow down deteriorates TCP throughput when packet losses are mainly due to corruption.

The second challenge is for TCP's error detection and recovery mechanism. High BER can cause a high amount of consecutive packet losses. Most commonly used TCP variants do not have the capability to efficiently recover from these losses. Either only one lost packet is recovered per Round Trip Time (RTT), or a timeout is needed to recover from substantial losses.

Our work differs from previous investigations of TCP in the respect that we investigate different combinations of existing congestion control and error detection strategies to find the combination providing the highest throughput. This is achieved by decomposing TCP into separate modules for congestion control and error detection as described in [5]. This approach allows us to determine if an update in congestion control or error detection has more impact on TCP performance.

The rest of the paper is organised as follows: Section 2 gives a short overview over the different congestion control and error detection mechanisms investigated in this paper. The following section presents throughput results for three different RTT values and increasing BER, and section 4 concludes the paper. 


\section{TCP'S CONGESTION CONTROL AND ERROR DETECTION MECHANISMS}

Since its initial standardisation, TCP went through several updates and changes to both its congestion control and its error detection mechanisms. The original TCP ([6]) did not include any congestion control at all and its error detection mechanism was purely timeout based. This TCP version is no longer used in the Internet and we do not consider it in our investigation.

The first TCP to include congestion control was TCP Tahoe ([7]). Its congestion control mechanism consists of two phases: Slow Start, which is executed at the beginning of the connection and after every packet loss, and Congestion Avoidance, which is entered once the connection has reached a certain transmission rate. The rate of data transmission in these two phases is governed by a congestion window (cwnd). Slow Start begins with a congestion window of one packet per Round Trip Time (RTT) and increases the window exponentially every RTT. During Congestion Avoidance the congestion window is increased linearly by one packet every RTT. We call this congestion control strategy TahoeCC. In addition to congestion control, TCP Tahoe also has a new mechanism to detect errors, which is called Fast Retransmit. Instead of waiting for a timeout, a threshold number of duplicate acknowledgment is taken as a packet loss indication (DupAckED).

TCP Reno ([8]) extends TCP Tahoe's congestion control by a Fast Recovery mechanism. Fast Recovery is entered only after Fast Retransmit. Instead of going back into Slow Start the congestion window is halved and new packets can be transmitted when duplicate acknowledgments arrive. When the acknowledgment for the retransmission is received, the connection goes straight into Congestion Avoidance (RenoCC). Error detection in TCP Reno is the same as for TCP Tahoe.

TCP NewReno ([9]) proposes alterations to TCP Reno's congestion control and error detection mechanisms. NewRenoCC alters the Fast Recovery mechanism by defining a Fast Recovery phase. This phase is entered after the first packet loss is detected with Fast Retransmit. It is only ended when the acknowledgment for the last packet transmitted before the loss was detected has been received. This allows TCP NewReno to recover from multiple losses without cuttings its congestion window several times. A new error detection mechanism, which we call NewRenoED, was developed to work together with NewRenoCC. NewRenoED takes partial acknowledgments as loss indications and retransmits the indicated packet immediately. A partial acknowledgment is an acknowledgment with a lower sequence number than the one of the last packet transmitted before the Fast Recovery phase is entered. 
The newest development for TCP is the use of selective acknowledgments ([10]) as error detection mechanism (SackED). Previously TCP receivers only acknowledged the highest in-order packet received. With selective acknowledgments, a receiver is able to acknowledge out-of-order packets. [10] only specifies the error recovery method and no congestion control mechanism. Theoretically, SackED should work well with any congestion control mechanism. It is expected that SackED will speed up TCP's error recovery compared to older error detection mechanisms.

In our study of TCP performance over links with high BER, all combinations of the existing TCP congestion control and error detection mechanisms were considered (TahoeCC, RenoCC, NewRenoCC, DupAckED, NewRenoED and SackED). We are interested in the combination of congestion control and error recovery to offer the highest throughput in a high BER environment.

The experiments were performed with the ns 2 simulator ([11]) using our own TCP implementation ([5]). The network consists of one sender and one receiver connected by a noisy link with a bandwidth of $1.5 \mathrm{Mbps}$. To show the impact that delay has on error recovery we considered three different RTTs in our experiments: 50 ms (metropolitan area network or low earth orbit (LEO) satellite), $200 \mathrm{~ms}$ (trans-Atlantic link, medium earth orbit satellite or multiple LEO hops) and $500 \mathrm{~ms}$ (geostationary earth orbit satellite). The default TCP Maximum Segment Size (MSS) of 536 bytes was chosen and the BER was varied from $10^{-8}$ to $10^{-4}$. The last two set of parameters result in packet error rates (PER) from 0 to $30 \%$ according to the following formula: $P E R=1-(1-B E R)^{M S S^{* 8}}$.

For all simulations, a fixed file size of $10 \mathrm{MB}$ was used, which is large enough to result in high throughput for most TCP variants ([4]) and small enough to achieve simulation results in a reasonable amount of time.

The following section discusses the throughput per RTT for increasing BER.

\section{THROUGHPUT RESULTS}

In this section, we present the throughput, in kilo Bytes $(\mathrm{kB})$ per second, achieved by the investigated TCP variants for increasing BER. The results for the three different RTT values are displayed in Figure 1 to Figure 3.

The most interesting observation from these results is that NewRenoED obtains the best as well as the worst performance, depending on the congestion control strategy, BER and RTT. The reasons for this behaviour will be discussed in the next two sub-sections. 
Throughput, RTT $50 \mathrm{~ms}$

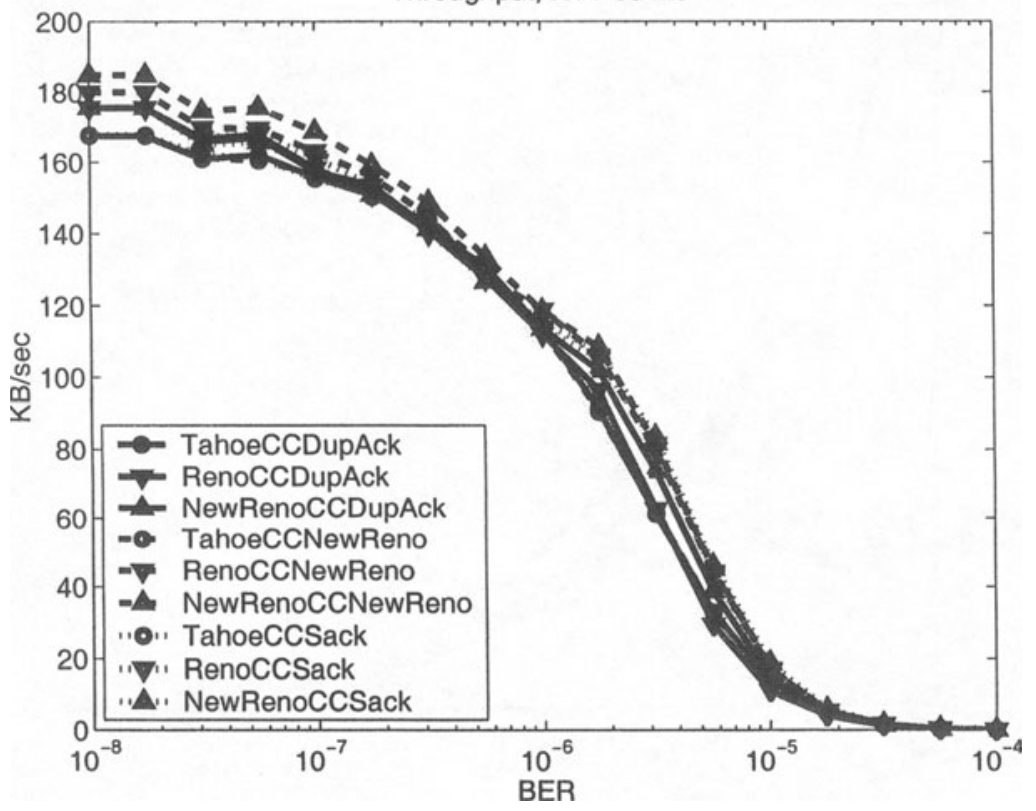

Figure 1. Throughput for a RTT of $50 \mathrm{~ms}$

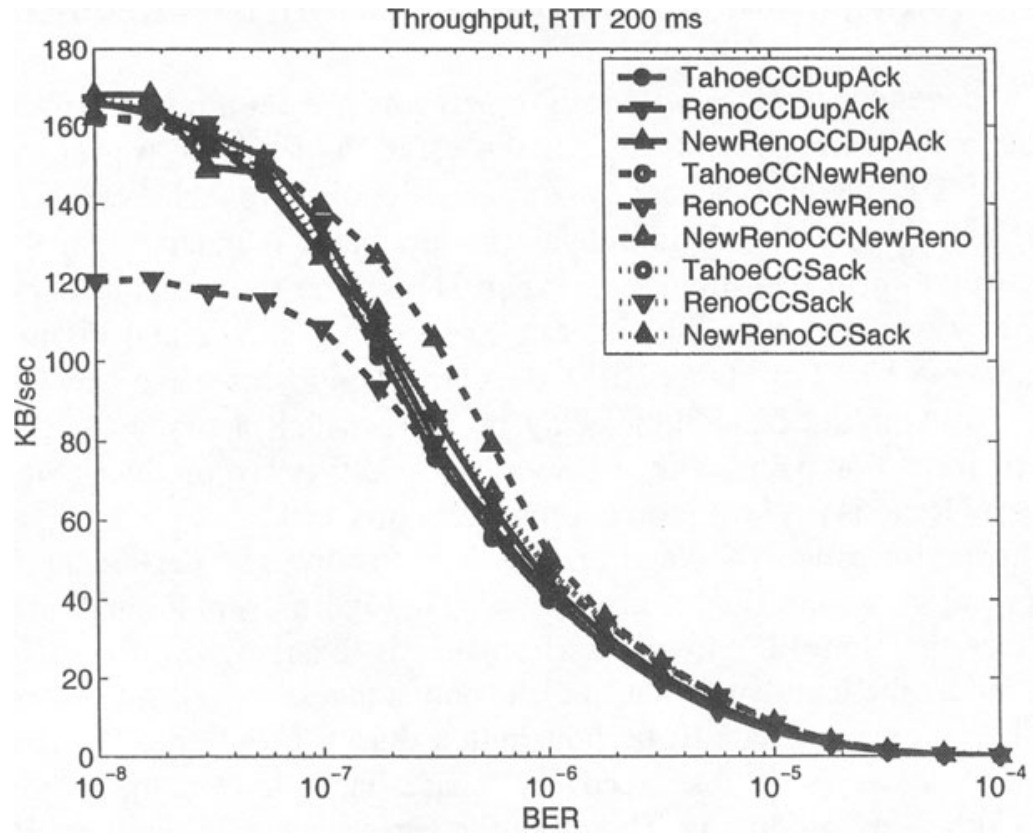

Figure 2. Throughput for a RTT of $200 \mathrm{~ms}$ 


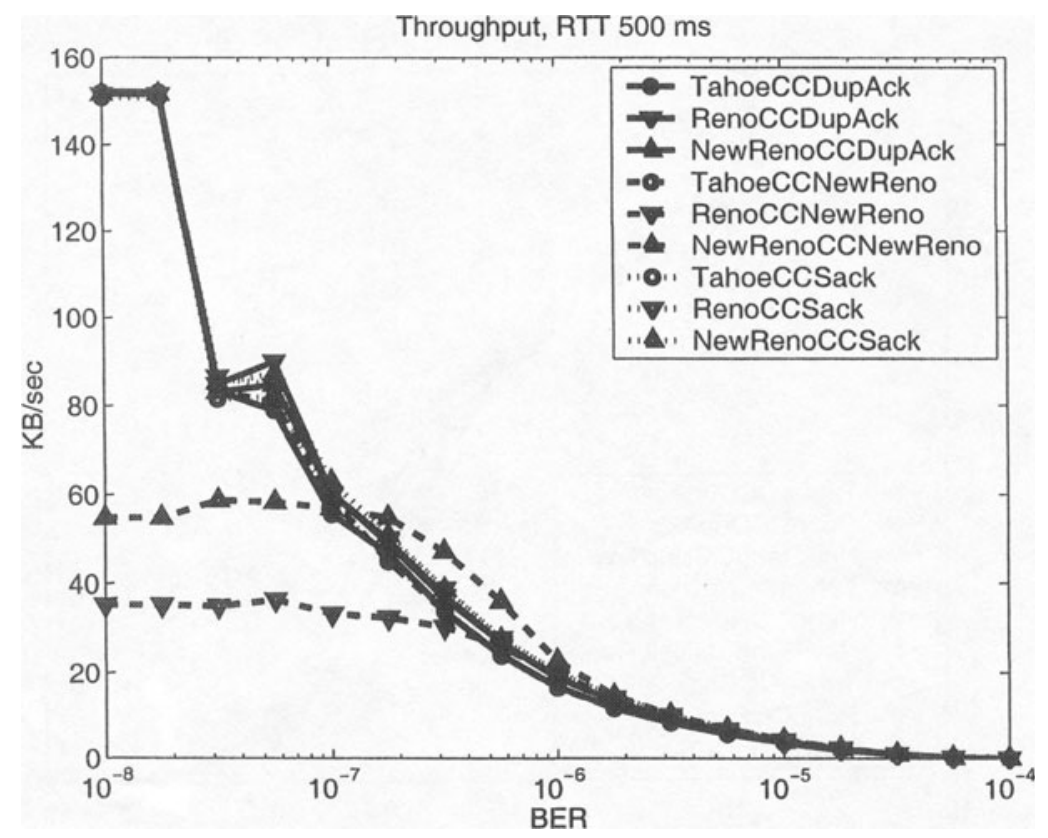

Figure 3. Throughput for a RTT of $500 \mathrm{~ms}$

\subsection{Reasons for worst throughput with NewRenoED}

The dependence of NewRenoED's performance on the bandwidth delay product and the file size was already discussed in [4]. There we showed that the minimal file size necessary for NewRenoED to achieve acceptable throughput for high bandwidth delay product paths is much larger than the $10 \mathrm{MB}$ chosen in our simulation experiments. The throughput degradation caused by our choice of file size can be seen in Figure 2 and Figure 3 for BERs below $10^{-7}$. For higher BERs the corruption losses make NewRenoED independent of the bandwidth delay product, which actually increases its performance. For paths with a lower bandwidth delay product (Figure 1) TCP NewReno is the best protocol for BERs up to $10^{-6}$.

The performance of NewRenoED is also strongly dependent on the accompanying congestion control strategy. In Figure 2 and Figure 3 it can be seen that NewRenoED's lowest performance is together with RenoCC. This is caused by the fact that RenoCC is the only congestion control strategy that does not allow new data to be transmitted during NewRenoED's long loss recovery phase. This loss recovery phase lasts longer the higher the bandwidth delay product is. Therefore the performance of NewRenoED and RenoCC gets worse when used over a path with high bandwidth delay 
product. NewRenoCC with NewRenoED (which is TCP NewReno) also has a low throughput when the bandwidth delay product is high but not as bad as NewRenoED with RenoCC. NewRenoCC can send new data packets during NewRenoED's transient phase, but when the file is too short, the performance is degraded.

\subsection{Reasons for best throughput with NewRenoED}

The most interesting property of NewRenoED is the fact that it gives the highest throughput results for BERs ranging from $10^{-6.75}$ to $10^{-5.75}$ independent of the bandwidth delay product. The reason for this can be seen in Figure 4. NewRenoED has two distinctive forms of detecting lost packets: three duplicate acknowledgments (dupacks), as all TCP error detection mechanisms, and partial acknowledgments. A recovery phase starts when a packet loss is detected with 3 dupacks. At this moment the highest sequence number sent is remembered and only when an acknowledgment covering this sequence number is received the recovery phase ends. During a phase, each additional loss is detected by a partial acknowledgment. The congestion window is only halved for the loss at the beginning of the recovery phase. For all other losses, it remains unchanged. Two very distinct recovery phases can be seen in Figure 4 The advantage of NewRenoED comes in the BER region when, on average, losses are not frequent enough to prematurely end the initial Slow Start phase. After Slow Start the long NewRenoED transient phase is entered. NewRenoCC is able to transmit new data packets during this transient phase, some of which get lost due to the high bit error rate. Once the first transient phase is finished, NewRenoED detects the first of these losses and enters a second transient phase. During this second transient phase, all corruption losses up to this point are recovered, while the congestion window is only halved once.

The higher the BER, the less likely it is for the initial Slow Start phase to be completed before a corruption loss occurs. In all three figures, In Figure 2 and Figure 3 this gradual drop in performance of NewRenoCC and NewRenoED can be noticed. For BERs above $10^{-5.75}$, the chance that Slow Start is completed successfully gets so low that NewRenoCC and SackED slightly outperforms NewRenoCC \& NewRenoED. 
TCP NewReno congestion window development

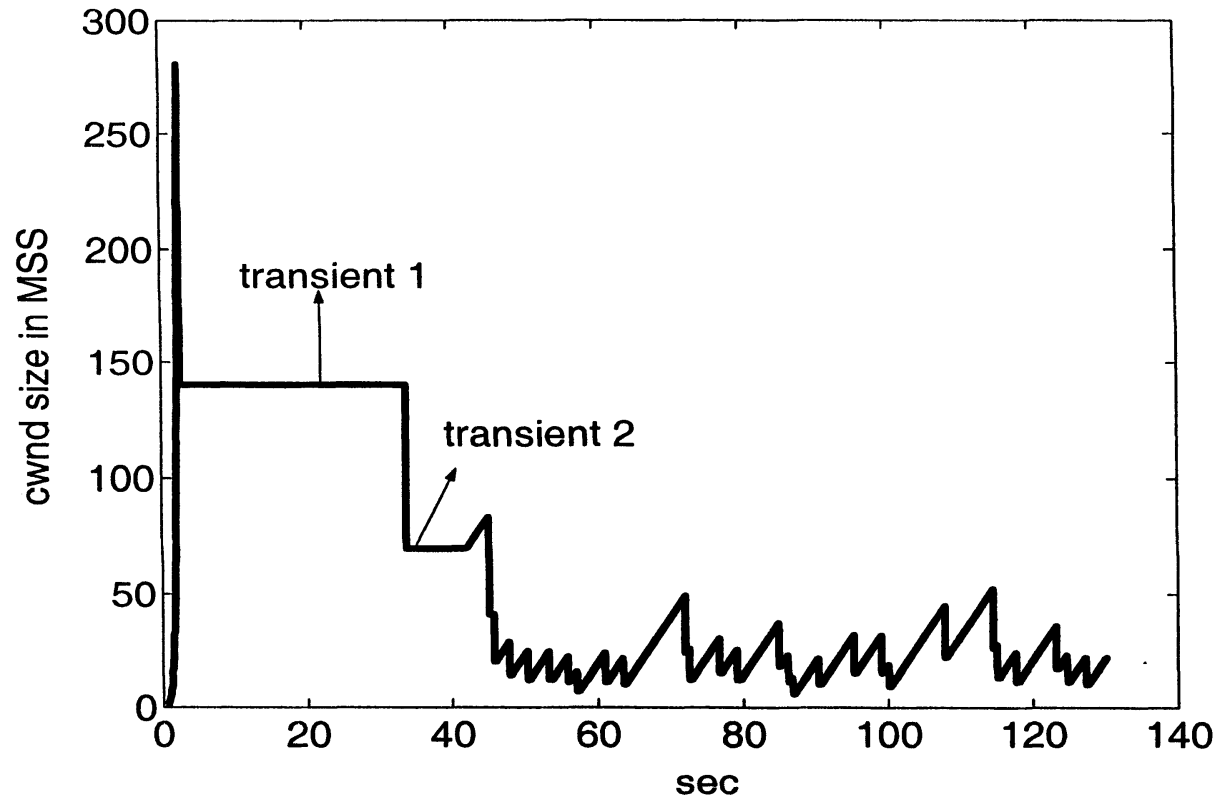

Figure 4. Congestion window for TCP NewReno

\subsection{Throughput difference between best and worst TCP}

Another interesting result from our simulation experiments is the similar performance between NewRenoED and SackED independent of the RTT and the combined congestion control strategy. SackED has been standardised because it was expected that the additional sack information would speed up the error recovery considerably. We were not able to confirm this with our experiments. SackED has a higher throughput than NewRenoED only for the lower RTT value of 50 ms and BERs higher than $10^{-6}$. Even then the throughput difference is less than 5\%. The difference in throughput between SackED and NewRenoED for the two higher RTTs and high BERs is even less. SackED only obtains better throughput for low BER values and high RTTs because the file size is too short to achieve a high throughput with NewRenoED.

To better illustrate the point that there is not much difference in throughput for all TCP variants we show the difference in throughput between all the investigated TCP variants in Figure 5 to Figure 7 . The maximal difference between the best and the worst protocol variant for any BER is displayed. RenoCC combined with NewRenoED has been excluded from the results with RTTs of $200 \mathrm{~ms}$ and $500 \mathrm{~ms}$ for BERs between $10^{-8}$ 
and $10^{-7}$ because of its exceptionally bad performance. NewRenoCC \& NewRenoED has been excluded for the same BER values from the $500 \mathrm{~ms}$ RTT graph, for the same reason.

The difference in throughput of the best and worst protocol is very much dependent on the RTT of the path. For a low RTT (Figure 5) the performance difference is below $10 \%$ for low BER. In the BER range from $10^{-6}$ to about $10^{-5.5}$ the choice of protocol has the most impact. The difference is negligible for higher BER.

The higher the RTT, the more the region with the highest performance difference shifts towards low BERs. For a RTT of $200 \mathrm{~ms}$ the peak in difference is between BERs $10^{-7}$ to $10^{-6.5}$. For BERs above and below these values, the difference is mostly below $5 \%$.

For the highest RTT in our investigation the maximal difference between TCP variants is around a BER of $10^{-7}$. The performance difference for the rest of BERs is even lower than for the other two RTT values.

This shows that most of the time it is hardly worth to talk about a best or a worst TCP variant. The small range of BERs for which the choice of protocol is important depends on the RTT of the connection, which is normally not known prior to the connection start. It is therefore difficult to chose the right protocol variant before the start of the connection.

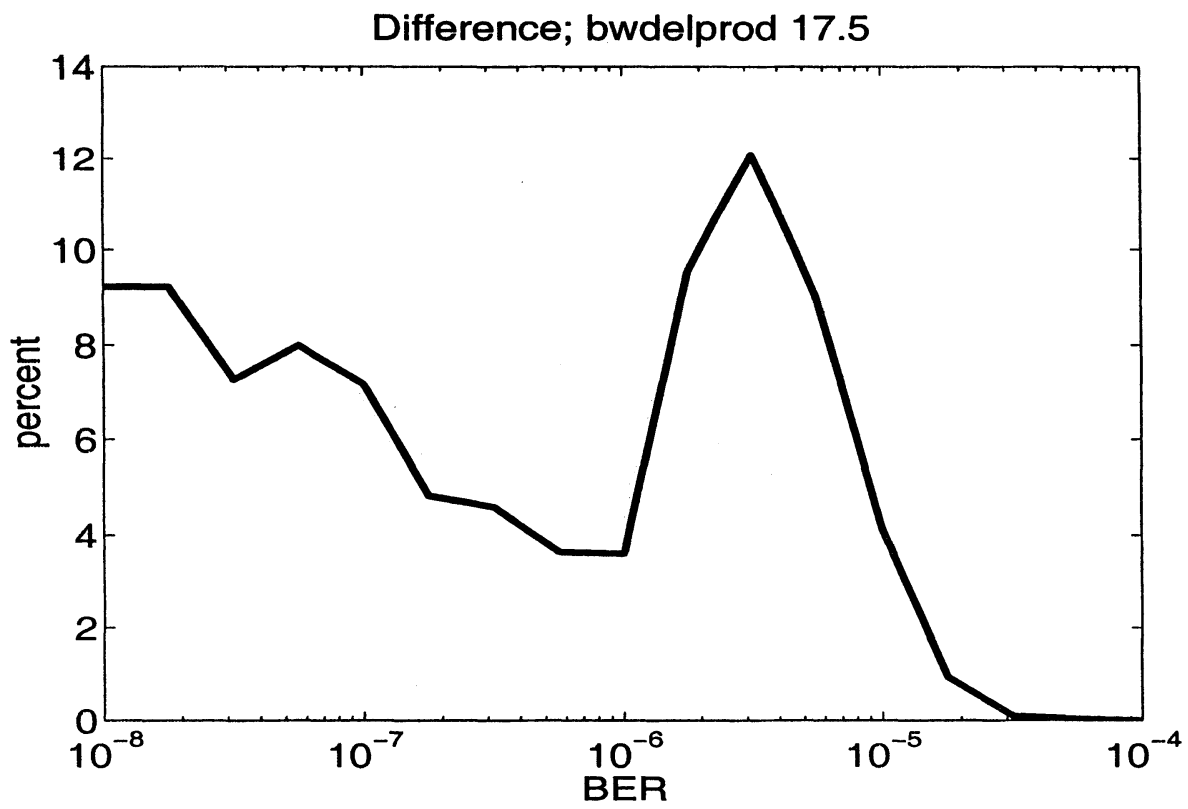

Figure 5. Throughput difference for $50 \mathrm{~ms}$ RTT 


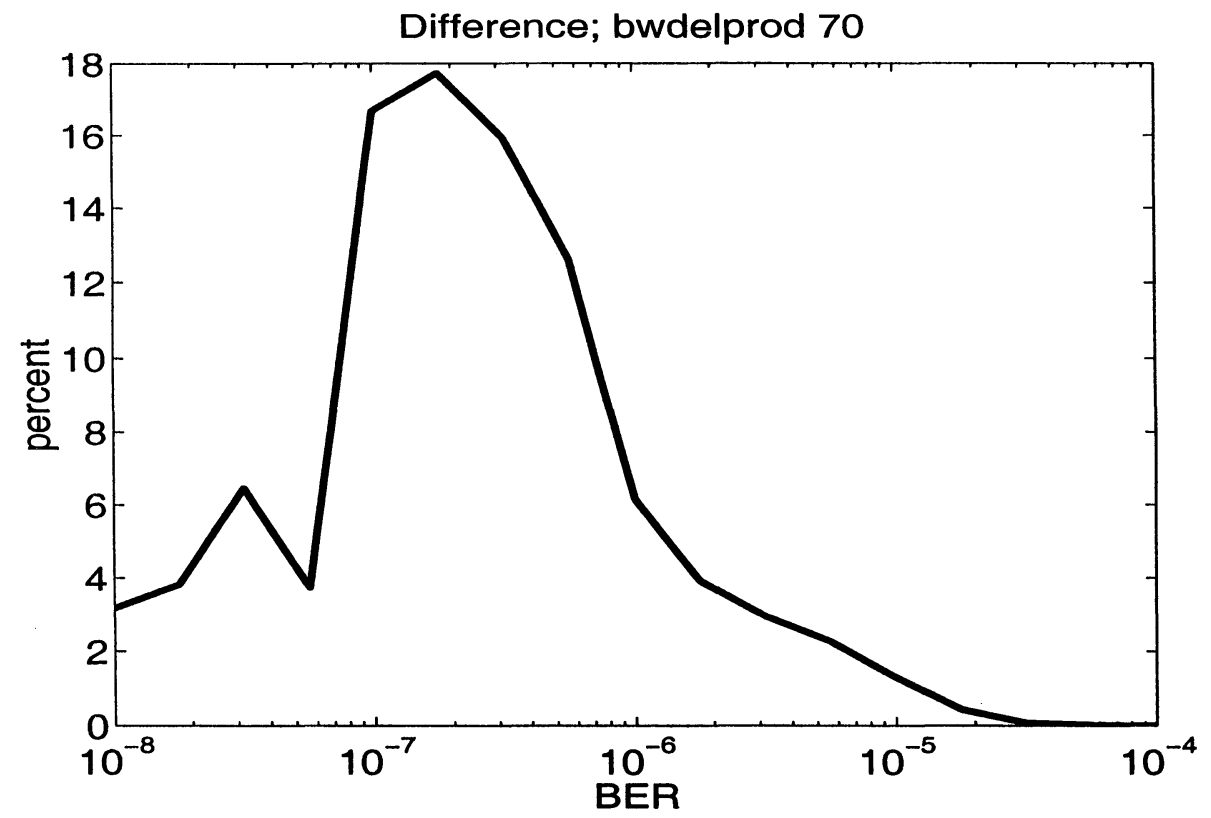

Figure 6. Throughput difference for $200 \mathrm{~ms}$ RTT

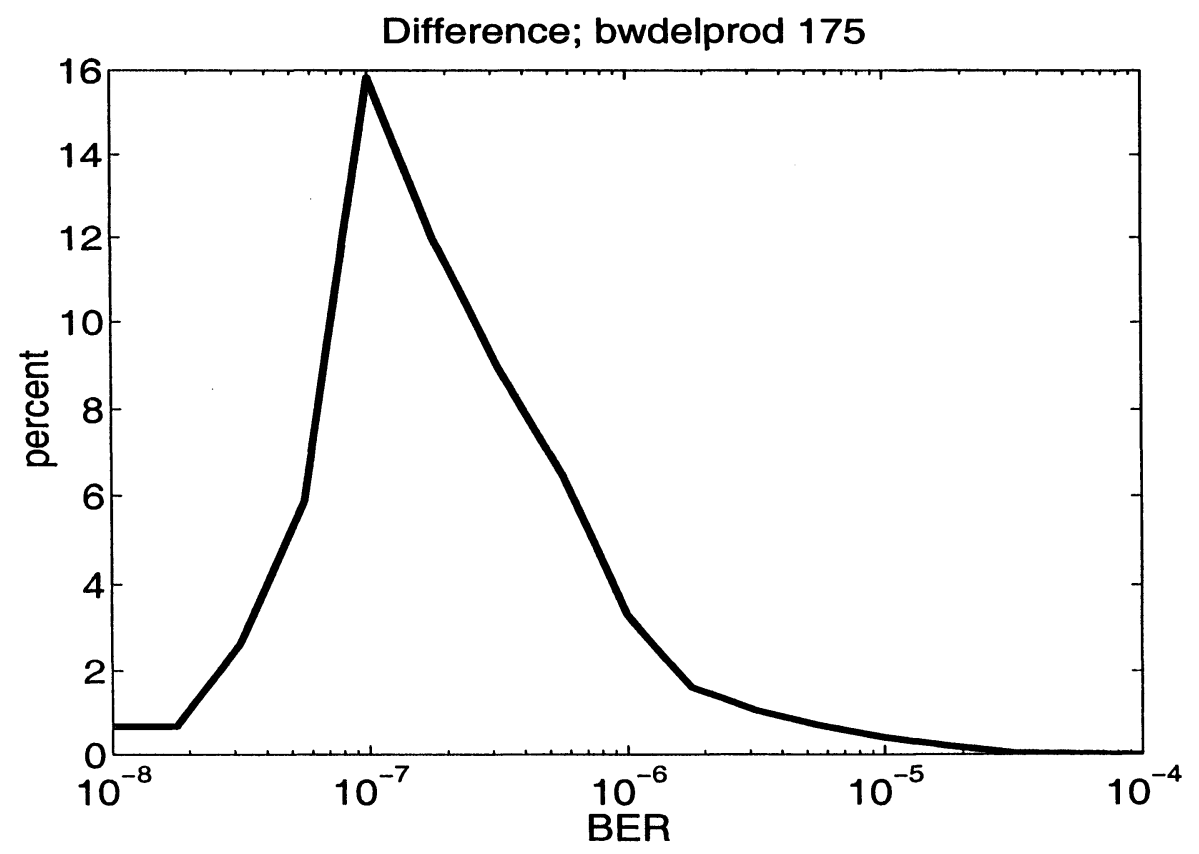

Figure 7. Throughput difference for $500 \mathrm{~ms}$ RTT 


\section{CONCLUSIONS}

In this paper, we investigated the throughput that a user can expect when TCP is used over links with a high BER. For this investigation, a new modular protocol implementation of TCP was used. This modular implementation allowed us to study the interaction between different proposed congestion control and error detection mechanisms.

We wanted to evaluate the impact that increasing BER and increasing RTT have on the performance of different TCP variants.

In general, we found that the difference in throughput between the different TCP variants is less than $10 \%$ for most BERs for all three RTT values considered. There was only a small range of BER values where the actual TCP variant chosen made an impact on the throughput. This range of BERs is dependent on the RTT.

The most important insight that we obtained was the similar throughput performance for NewRenoED and SackED together will all congestion control strategies. SackED is a more complex error detection mechanism that involves co-operation from the receiver to indicate out-of-order packets. The expectation was that this more complex mechanism should bring a considerable performance benefit for error recovery. Our results did not confirm this. If the file size is large enough, NewRenoED performs better than SackED for low BERs and almost as well as SackED for high BERs. NewRenoED achieves this performance with no co-operation from the receiver. We therefore suggest the usage of TCP NewReno is sufficient for high BER environments and it is not necessary to update to TCP Sack.

\section{ACKNOWLEDGMENTS}

This work was carried out with financial support from the Commonwealth of Australia through the Cooperative Research Centres Program.

\section{REFERENCES}

[1] V.G. Bharadwaj, J.S. Baras, N.P. Butts, "Internet Service via Broadband Satellite Networks", CSHCN TR 99-11 Center for Satellite and Hybrid Communication Networks Technical Report, http://www.isr.umd.edu/TechReports/CSHCN/, 1999

[2] R.C. Durst, G.J. Miller, E.J. Travis, "TCP extensions for space communications", Wireless-Networks.vol.3, no.5; 1997; p.389-403

[3] N. Ghani, S. Dixit "TCP/IP enhancements for satellite networks", IEEE-CommunicationsMagazine.vol.37, no.7; July 1999; p.64-72 
[4] T.Lang, D.Floreani, "On the achievable throughput of different TCP variants over high bandwidth delay product paths", submitted to Globecom 2002 http://www.itr.unisa.edu.au/ -tanja

[5] T.Lang, D.Floreani, "Modular TCP Design and Its Application in Performance Evaluation of Different TCP Versions for Wireless Environments", Sixth IEEE Symposium on Computers and Communications (ISCC 2001), July 3-5, 2001, Tunisia.

[6] J.Postel, "Transmission Control Protocol, STD 7, RFC 793", Internet Request for Comments 793, Sept 1981

[7] V. Jacobson, "Congestion Avoidance and Control", Computer Communication Review, vol. 18, no. 4, pp. 314-329, Aug. 1988. ftp://ftp.ee.lbl.gov/papers/congavoid.ps.Z.

[8] M. Allman, V. Paxson, W. Stevens, "TCP Congestion Control, RFC 2581", Internet Request for Comments 2581, April 1999

[9] S. Floyd, T. Henderson, "The NewReno Modification to TCP's Fast Recovery Algorithm, RFC 2582”, Internet Request for Comments 2582, April 1999.

[10] M. Mathis, J. Mahdavi, S. Floyd, A. Romanow, "TCP Selective Acknowledgment Options, RFC 2018", Internet Request for Comments 2018, Oct 1996

[11] Network Simulator version 2, http://www.isi.edu/nsnam/ns/ 